\title{
Harmonic Frequency Estimation Algorithm based on ESPRIT and MSWF in Power System
}

\author{
Sun Xiangwen", Cao Zhe and Tian Wei \\ School of Electrical Engineering, Henan University of Science and Technology, Luoyang, 471023, P.R. China
}

\begin{abstract}
This paper points out the deficiency in the current harmonic frequency estimation algorithm in power system. In order to improve the accuracy of detection and reduce the computational complexity, the study combined the ESPRIT algorithm with multistage Wiener filter (MSWF) recurrence to achieve fast estimation of harmonic frequency. Theoretical analysis and simulation experiment show that the algorithm had relatively low requirement for the amount of data, and demonstrated good frequency resolution characteristics and anti-jamming capability, which made it ideally suitable for harmonic analysis in power system.
\end{abstract}

Keywords: ESPRIT, harmonic frequency estimation, MSWF.

\section{INTRODUCTION}

For power system of $50 \mathrm{~Hz}$ power frequency, its frequency spectrum can be obtained by Fourier analysis of voltage and current. In general, frequency spectrum component with frequency of integer multiple of $50 \mathrm{~Hz}$ spectral is called harmonics. It is known that when the harmonics' components in the power grid exceed the limitation standard, security, reliability of the power system and electrical equipment operation are seriously affected. Therefore, currently, speedy detection method of harmonics has become an objective for scholars at home and abroad who are committed to study the detection methods and a number of achievements have been made, such as fast Fourier transform (FFT) [1], singular value decomposition (SVD) [2], wavelet transform (Wavelet) [3], artificial neural network (ANN) [4], and multiple signal classification (MUSIC) algorithm [5].

FFT is the most widely applied in current harmonic frequency estimation. Theoretically, when synchronous sampling conditions are met, FFT algorithm can obtain accurate signal spectrum, and thus calculate the value of harmonic frequency, amplitude and phase. However, as the actual grid frequency often fluctuates in the frequency accessory, which leads to non-synchronous sampling, the resulting spectrum leakage may produce false interharmonics, or hide real inter-harmonics, thus limiting FFT application [5] for accurate detection of inter-harmonics. Although windowed interpolation method can be adapted to correct FFT detection result, the correction process has relatively strict requirement for the windowing type and the window function width, including relatively large computation burden, however, it is difficult to meet the requirements [6] of real-time analysis.

Wavelet transform [7] has a good localization characteristic, but because of the presence of aliasing in the

*Address correspondence to this author at the School of Electrical Engineering, Henan University of Science and Technology, Luoyang, 471023, P.R. China; Tel: +86 15670318675;

E-mail: sun_xiangwen@163.com frequency domain of different wavelet functions, interharmonic frequency detection lacks accuracy. Harmonic frequency estimation algorithm based on artificial neural network requires a lot of prior information and network training. In addition, due to high computational complexity and poor timeliness, it is unable to meet the needs of realtime harmonic detection.

MUSIC method is a parameter estimation algorithm based on subspace characteristic decomposition, which is widely used in array signal processing and DOA estimation. The method takes full advantage of orthogonality of signal subspace and noise subspace and achieves estimation of unknown parameter by spectrum peak search of pseudospatial spectrum, thus demonstrating high estimation accuracy and good statistical property [8].

However, this method requires calculation of sample covariance matrix and characteristic decomposition. Meanwhile, this method also requires point-by-point computation of pseudo-spatial spectrum in the full frequency domain in comparison to search spectrum peak. If there is a high requirement for estimation accuracy, stepping distance in the search should not be too large, otherwise, it will lead to the picket fence effect. Therefore, the computational complexity of the method is very high.

To solve the above problem, this paper proposed a new harmonic frequency estimation algorithm in power system. Firstly, the algorithm completed decomposition of signal subspace and estimation of harmonic number through MSWF recurrence, which avoided sample covariance matrix characteristic decomposition required by MUSIC algorithm. Secondly, it used estimation of signal parameters via rotational invariance techniques (ESPRIT) and completed estimation of harmonic frequency, which avoided point-bypoint computation of pseudo-spatial spectrum in the full frequency domain to search spectrum peak in MUSIC algorithm. Therefore, compared with standard MUSIC algorithm, computational complexity of harmonic frequency estimation algorithm proposed in this paper has been significantly reduced. 
This paper discussed the above-mentioned issues. Firstly, it describesd the harmonic frequency estimation principle based on ESPRIT algorithm; secondly, it introduced the technique for subspace rapid decomposition via MSWF recurrence; thirdly, it discussed the estimation method of harmonic number $\mathrm{M}$; finally, it verified the harmonic frequency estimation algorithm proposed in this paper via numerical simulation experiment.

\section{SIGNAL MODEL}

For power system of $50 \mathrm{~Hz}$ frequency, the frequency spectrum can be obtained after Fourier decomposition of voltage and current. In general, frequency spectrum component with frequency of integer multiple of $50 \mathrm{~Hz}$ spectral is called harmonics. Therefore, sampling signal of voltage and current signals with noise, frequency, and harmonics in the power system can be expressed as:

$x(n)=\sum_{i=1}^{M} A_{i} \cos \left(2 \pi f_{i} n+\phi_{i}\right)+e(n)$

where, $x(n)$ is the sampling signal, $A_{i}$ is the harmonic amplitude, $f_{i}$ is normalized harmonic frequency, $n$ is the sampling time, $\phi_{i}$ is the initial phase for harmonics, $e(n)$ represents noise, and $M$ is the number of harmonics. To facilitate the process, $x(n)$ was transformed into complex frequency signal. Following this, equation (1) can be rewritten as:

$x(n)=\sum_{i=1}^{M} A_{i} e^{j\left(2 \omega_{i} n+\phi_{i}\right)}+e(n)$

Notate:

$$
\begin{aligned}
& \mathbf{X}(\mathbf{n})=[x(n), x(n-1), \mathrm{L}, x(n-K \quad 1)]^{T} \\
& \mathbf{Y}(\mathbf{n})=[x(n+1), x(n), \cdots, x(n-K+2)]^{T} \\
& S(n)=\left[\begin{array}{llll}
A_{1} e^{j\left(n \omega_{1}+\varphi_{1}\right)} & A_{2} e^{j\left(n \omega_{2}+\varphi_{2}\right)} & \cdots & A_{M} e^{j\left(n \omega_{M}+\varphi_{M}\right)}
\end{array}\right]^{T} \in C^{M \times 1} \\
& E(n)=\left[\begin{array}{llll}
e(n) & e(n+1) & \cdots & e(n+M-1)
\end{array}\right]^{T} \in C^{M \times 1} \\
& \Phi=\operatorname{diag}\left\{e^{j \omega_{1}}, e^{j \omega_{2}}, \cdots e^{j \omega M}\right\} \in C^{M \times M} \\
& A=\left[\begin{array}{cccc}
1 & 1 & \cdots & 1 \\
e^{-j \omega_{1}} & e^{-j \omega_{2}} & \cdots & e^{-j \omega_{M}} \\
\vdots & \vdots & \cdots & \vdots \\
e^{-j K \omega_{1}} & e^{-j K \omega_{2}} & \cdots & e^{-j K \omega_{M}}
\end{array}\right] \in C^{K \times M}
\end{aligned}
$$

After introduction of the above notation, equation (2) can be expressed as: $\mathbf{X}(\mathbf{n})=A S(n)+E(n)$

Equation (4) can be expressed as:

$$
\begin{aligned}
& \mathbf{Y}(\mathbf{n})=\mathbf{X}(\mathbf{n}+1)=A S(n+1)+E(n+1) \\
& \quad=\mathrm{A} \Phi S(n+1)+E(n+1)
\end{aligned}
$$

\section{FREQUENCY ESTIMATION PRINCIPLE BASED ON ESPRIT ALGORITHM}

The auto-correlation $\mathbf{R}_{\mathbf{X}}$ of $\mathbf{X}(\mathbf{n})$ and the crosscorrelation matrix $\mathbf{R}_{\mathrm{XY}}$ of $\mathbf{X}(\mathbf{n}), \mathbf{Y}(\mathbf{n})$ :

$$
\begin{aligned}
& R_{X}=E\left\{X(n) X^{H}(n)\right\}=A P A^{H}+\sigma^{2} I \\
& R_{X Y}=E\left\{X(n) Y^{H}(n)\right\}=A P \Phi^{H} A^{H}+\sigma^{2} Z
\end{aligned}
$$

where, $\sigma^{2}$ is the white noise power,

$$
\mathrm{Z}=\left[\begin{array}{cccc}
0 & 1 & & 0 \\
& 0 & \ddots & \\
& & \ddots & 1 \\
0 & & & 0
\end{array}\right] P=E\left\{S(n) S^{H}(n)\right\} .
$$

Structure matrix beam $\left(C_{\mathrm{xx}}, C_{\mathrm{XY}}\right)$, where: $C_{\mathrm{Xx}}=\mathrm{APA}^{\mathrm{H}}$ , and $C_{\mathbf{X Y}}=\mathrm{AP} \Phi^{\mathrm{H}} \mathrm{A}^{\mathrm{H}}$. Following this, it can be proved that $e^{j \omega_{i}}$ is generalized eigenvalue of matrix beam $\left(C_{\mathbf{X X}}, C_{\mathbf{X Y}}\right)$. Therefore, harmonic frequency estimation can be obtained by extracting generalized eigenvalue of $\left(C_{\mathrm{XX}}, C_{\mathrm{XY}}\right)$ at unit circle. Operating principle of ESPRIT algorithm reveals that, compared with MUSIC algorithm, estimation of harmonic frequency with this algorithm avoided point-by-point computation of pseudo-spatial spectrum in the full frequency domain to search spectrum peak, thus greatly reducing calculation amount. Especially, if the requirement for estimation accuracy is high, this gap becomes more apparent.

\section{FAST SUBSPACE DECOMPOSITION BASED ON MSWF}

As mentioned above, application of ESPRIT algorithm in harmonic frequency estimation needs characteristic decomposition of sample autocorrelation matrix and matrix beam. The computational complexity is high and thus it is not conducive to real-time processing. Therefore, there is a need for a methodwith reduced computational complexity to obtain signal characteristic subspace and thereby reduce time cost of this process. Many scholars have done a lot of research in this regard and have made many valuable results, such as QR iteration, power iteration algorithm, conjugate gradient algorithm, and Lanczos iteration algorithm. These methods avoid characteristic decomposition of sample covariance matrix, and can reduce computational complexity to a certain extent. However, they still need calculation of sample covariance matrix and the computational complexity is still relatively high.

MSWF is a realization form of nested Wiener filter put forward by Goldstein in 1997. Without calculation of sample covariance matrix and characteristic decomposition, this method can obtain signal subspace and noise subspace, with characteristics [9] of small computation amount and fast convergence.

The basic steps of MSWF algorithm based on related subtraction structure are as follows [10]: 
1. N-point sampling data is used and data array $X$ is constructed in accordance with equation (3)

Define training signal $d_{0}(k)=e_{1}^{T} x_{1}(k+\tau), \quad$ and observation signal $X_{0}=\left[\mathrm{x}_{2}, \mathrm{x}_{3}, \cdots, \mathrm{x}_{p}\right]^{T}$.

2. M times forward recurrence is:

FOR $i=1,2, \cdots, M$

$h_{i}=E\left[d_{i-1}^{*}(k) X_{i-1}(\mathrm{k})\right] /\left\|E\left[d_{i-1}^{*}(k) X_{i-1}(\mathrm{k})\right]\right\|_{2}$

$d_{i}(k)=h_{i}^{\mathrm{H}} X_{i-1}(k)$

$X_{i}(k)=X_{i-1}(k)-h_{i} d_{i}(k)$

Consider $\mathrm{m}$ dimensional Krylov subspace $\kappa^{m}\left(R_{x}, f\right)=\operatorname{Span}\left\{f, R_{x} f, \cdots, R_{x}{ }^{m-1} f\right\}$ composed of $R_{x} \in C^{N \times N}$ and any vector $f \in C^{N \times 1}$. Considering equation (5), shift in invariant property of Krylov subspace derives that:

$$
\begin{aligned}
\kappa^{m} & \left(R_{x}, f\right)=\kappa^{m}\left(R_{x}-\sigma_{n}^{2} I_{N}, f\right) \\
& =\kappa^{m}\left(V_{s} \sum_{s} V_{s}^{H}+\sigma_{n}^{2} V_{n} V_{n}^{H}-\sigma_{n}^{2} I_{N}, f\right) \\
& =\kappa^{m}\left(V_{s}\left(\sum_{s}-\sigma_{n}^{2} I\right) V_{s}^{H}, f\right)
\end{aligned}
$$

From equation (7), it can be observed that if $f \in \operatorname{span}\left\{V_{s}\right\}, \kappa^{m}\left(R_{x}, f\right)$ is equivalent to the signal subspace. At this time, a group of basis of $\kappa^{m}\left(R_{x}, f\right)$ is requested, which can be expanded into $V_{s}$.

Reference $[9,10]$ has proven that, when $e(n)$ is empty white noise, $d_{0}(k)=e_{1}^{T} x_{0}(k+\tau) \in \operatorname{span}\left\{V_{s}\right\}$ Therefore, $\kappa^{m}\left(R_{x}, d_{0}(k)\right)$ is equivalent to the signal subspace $V_{s}$.

Meanwhile, according to MSWF theory, $\left\{h_{1}, h_{2}, \cdots h_{M}\right\}$ obtained by M times, MSWF forward recurrence is a set of standard orthogonal basis of $\kappa^{m}\left(R_{x}, d_{0}(k)\right)$. Therefore, MSWF can be used for rapid calculation of signal subspace.

It should be stressed that the computational complexity of MSWF forward recurrence to calculate signal subspace $V_{s}$ is about $O\left(M P^{2}\right)$. However, computational complexity of sample covariance matrix calculation and eigenvalue decomposition is $O\left(M P^{2}+P^{3}\right)$. Therefore, computation of signal subspace $V_{s}$ with MSWF forward recurrence can significantly reduce calculation amount of algorithm.

\section{ESTIMATION OF SIGNAL NUMBER}

As mentioned above, before application of MSWF to obtain signal subspace, dimension $M$ of signal subspace needs to be obtained. In theory, when the true covariance matrix $R_{x}$ is known, $\mathrm{M}$ can be obtained through its characteristic decomposition. However, in practice, true covariance matrix is not available, and only its approximate estimation $\widetilde{R_{x}}$ can be obtained Therefore, it is necessary to use other effective methods to estimate the number $M$ of harmonics.

Currently, the main methods used to estimate the signal subspace dimension are AIC criterion and MDL criterion based on information theory, gerschgorin disk method, smooth rand profile, and canonical correlation method, of which, AIC and MDL are the most common methods to estimate subspace dimension in white noise environment.

In general, basic principles of methods AIC and MDL involve using eigenvalue of $\widetilde{R_{x}}$ to determine cost function $J(k)$, based on consistent estimate of $M$, and estimate phenomenon based on this method, for obtaining estimate of signal subspace dimension $M$ by minimizing $J(k)$. In comparison, AIC criterion is not used when sampling number is relatively small, but MDL provides consistent estimate of $M$.

Examining standard MDL criterion for subspace dimension estimation:

$$
\begin{aligned}
M D L(k)=L(k)+ & P(k) \\
& =-\ln \left(\frac{\prod_{i=k+1}^{M} \lambda_{i}^{\frac{1}{M-k}}}{\frac{1}{M-k} \sum_{i=k+1}^{M} \lambda_{i}}\right)^{(M-k) N}+\frac{k}{2}(2 M-k) \ln N
\end{aligned}
$$

Apparently, $L(k)$ in equation (8) is related to geometric mean value of the rear $(M-k)$ eigenvalues of $\widetilde{R_{x}}$ and the value of arithmetic mean.

From equation(5), it can be observed that, based on matrix theory, if the real number of signal source is $D$, then $\widetilde{R_{x}}$ should have $D$ non-zero eigenvalues, denoted by descending order, $\lambda_{1}, \lambda_{2} \ldots \lambda_{D}$. Among the remaining $M$ - $D$ eigenvalues, $\lambda_{D+1}, \lambda_{D+2}, \cdots, \lambda_{M}$ is the minimum and equal. (AUTHOR: Please clarify the highlighted) Thus, the size relationship of the above $\mathrm{M}$ eigenvalues under high SNR condition is:

$\lambda_{1}>\lambda_{2} \cdots>\lambda_{D}>\lambda_{D+1}=\lambda_{D+2}=\cdots=\lambda_{M}=\sigma^{2}$

Under this condition, by considering the relationship between arithmetic mean and the geometric mean, it can be observed that:

$L(k) \geq 0$, if and only if $k=D, L(k)=0$.

Meanwhile, $P(k)$ is the quadratic function of $k$ which apparently monotonically increases when $k \in[0, M-1]$. There-fore, equation (8) acquires minimum value when $k=$ D.

At low SNR condition, equation (9) becomes false. Use of MDL at this time will produce error estimation of signal subspace dimension. However, taking into account that in actual power system, noise amplitude is about $0-1 \%$ of fundamental harmonic signal amplitude, and SNR is relatively high $(30 \mathrm{~dB})$, it is relatively appropriate to estimate harmonics number in the power system with this criterion. 
Obviously, if signal subspace dimension is estimated with MDL criterion, calculation of sample covariance matrix $\widetilde{R_{x}}$ and characteristic decomposition are needed, which eliminate the cause of using MSWF algorithm to reduce the computational complexity. Literature [9] has demonstrated that, in the absence of covariance matrix eigenvalues, $L(k)$ in MDL criterion converges with probability 1 at

$$
-\ln \left(\frac{\prod_{i=k+1}^{M} \sigma_{d_{i}}^{2 \frac{1}{M-k}}}{\frac{1}{M-k} \sum_{i=k+1}^{M} \lambda_{i}}\right)^{(M-k) N}
$$

where, $\sigma_{d_{i}}^{2}=E\left(\left|d_{i}\right|^{2}\right)$. Therefore, only by using the information obtained via MSWF recurrence, estimation of signal subspace dimension $M$ can be obtained, thereby avoiding cost brought by characteristic decomposition of $\widetilde{R}_{x}$.

In summary, the harmonic frequency estimation algorithm in power system proposed in this paper is as follows:

1. MSWF forward recurrence is applied. With the recursive result, the improved MDL criterion is combined with the estimated signal subspace dimension $\mathrm{M}$.

2. MSWF recurrence to complete the decomposition of $\mathbf{R}_{\mathbf{X}}$ is used Its minimum eigenvalue is taken as the estimate of noise power $\sigma^{2}$.

3. Matrix beam $\left(C_{\mathrm{Xx}}, C_{\mathrm{XY}}\right)$ is structured and MSWF is used to complete its generalized characteristic decomposition.

4. Characteristic root of $\left(C_{\mathrm{Xx}}, C_{\mathrm{XY}}\right)$ is used on the unit circle to complete harmonic frequency estimation.

\section{SIMULATION EXPERIMENT}

\section{Experiment 1}

The simulation analysis used each measured harmonic parameter of line voltage $V_{b c}$ of $\mathrm{Xi}$ 'an $35 \mathrm{kV}$ steel busbar, with phase position to be self-designed. The simulation signals are as follows:

$$
\begin{gathered}
x(t)=37.66 \cos (2 \pi \times 50 t+\pi / 4)+0.933 \cos (2 \pi \times 100 t+\pi / 36) \\
+1.813 \cos (2 \pi \times 150 t+\pi / 18)+0.885 \cos (2 \pi \times 200 t+\pi / 12) \\
+1.943 \cos (2 \pi \times 250 t+\pi / 9)+0.97 \cos (2 \pi \times 300 t+\pi / 8)
\end{gathered}
$$

In this experiment, sampling frequency was $f_{s}=1000 \mathrm{~Hz}$, test time was $30 \mathrm{~ms}$. The resulting sequence of samples was estimated to test the effect of the proposed estimation algorithm. The result is shown in Fig. (1).

For comparison, under the same condition, FFT was used to conduct spectrum estimation of the resulting sequence of samples and the result is shown in Fig. (2).
Analyzing the experimental results, even in the absence of noise and when the sampling frequency is an integer multiple of the fundamental frequency, if the amplitude of the harmonic component is low and the amount of sample data is small, estimation using FFT may miss some harmonic components. Specifically, FFT spectrum of Fig. (2) only has three distinct spectral peaks, approximately corresponding to three harmonics of $50 \mathrm{~Hz}, 150 \mathrm{~Hz}$, and $250 \mathrm{~Hz}$. However, for comparison, the algorithm accurately estimated six harmonic components (corresponding to 6 characteristic roots in circle unit in Fig. (1)) in the detection signals.

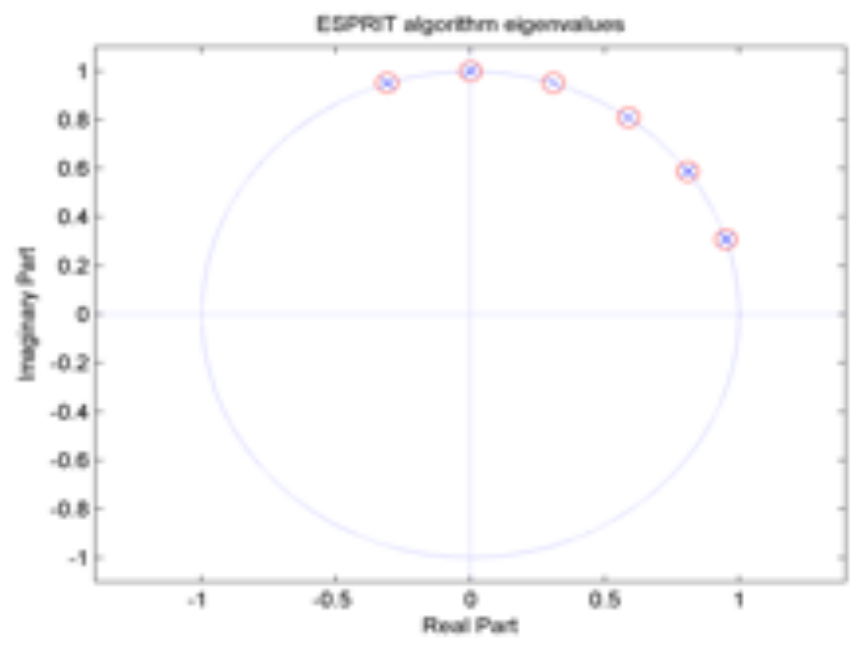

Fig. (1). Test 1 time series analysis result.

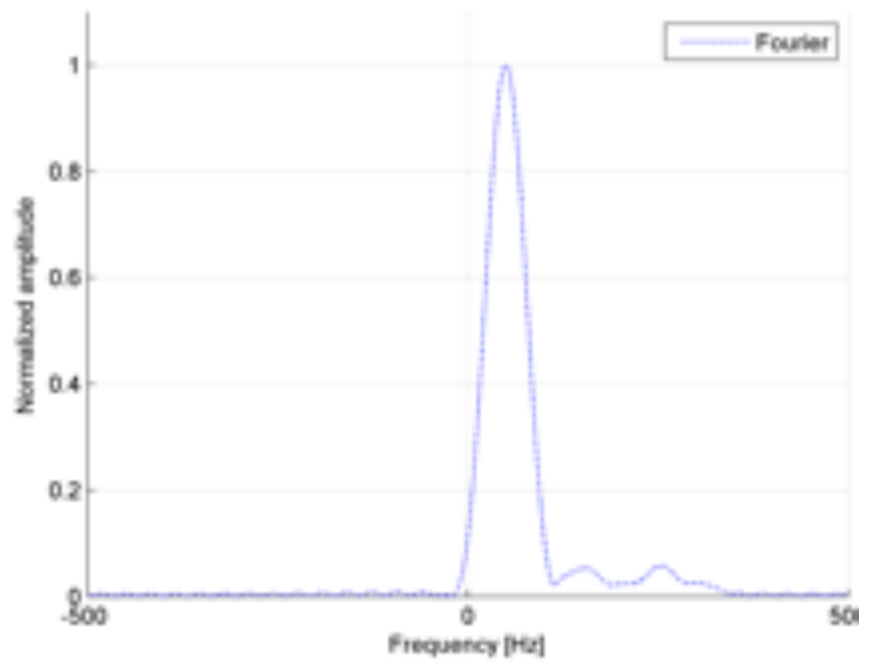

Fig. (2). FFT analysis result.

\section{Experiment 2}

To verify the performance of the algorithm in a noisy environment, this algorithm was used based on experiment 1 . Continuously changing SNR from 10 to 80 , the above experiment was repeated 100 times at each SNR, and the covariance of each harmonic estimate was calculated to validate the performance of the algorithm.

From the experimental results, it was observed that when SNR is small, the performance of this algorithm will decline to a certain degree, but when SNR increases, the estimated 
Table 1. Harmonic frequency test result of experiment 2.

\begin{tabular}{|c|c|c|c|c|c|c|c|c|}
\hline SNR & $\mathbf{1 0}$ & $\mathbf{2 0}$ & $\mathbf{3 0}$ & $\mathbf{4 0}$ & $\mathbf{5 0}$ & $\mathbf{6 0}$ & $\mathbf{7 0}$ & $\mathbf{8 0}$ \\
\hline \hline 1 & 0 & 0 & 0 & 0 & 0 & 0 & 0 & 0 \\
\hline 2 & 3.9111 & 0 & 0 & 0 & 0 & 0 & 0 & 0 \\
\hline 3 & 0.7149 & 0.0316 & 0 & 0 & 0 & 0 & 0 \\
\hline 4 & 10.4201 & 0 & 0 & 0 & 0 & 0 & 0 \\
\hline 5 & 0.0304 & 0 & 0 & 0 & 0 & 0 & 0 & 0 \\
\hline 6 & 0.0304 & 0 & 0 & 0 & 0 & 0 & 0 \\
\hline
\end{tabular}

covariance value of each harmonic frequency decreases rapidly. When SNR is greater than 30 , it will be reduced to zero, and then stops changing. By considering that noise amplitude of actual power system is about $0-1 \%$ of fundamental signal amplitude, SNR is relatively high $(30 \mathrm{~dB})$. Therefore, this algorithm proved to be suitable for application in harmonic frequency estimation in power system (Table 1).

\section{Experiment 3}

$$
\begin{aligned}
x(t)= & 0.3 \cos \left(2 \pi \times 37 t+70^{\circ}\right)+0.7 \cos \left(2 \pi \times 50 t+80^{\circ}\right) \\
& +1.0 \cos \left(2 \pi \times 87 t+30^{\circ}\right)+0.5 \cos \left(2 \pi \times 88.66 t+90^{\circ}\right) \\
+ & 0.4 \cos \left(2 \pi \times 150 t+40^{\circ}\right)
\end{aligned}
$$

The sample sequence contains three inter-harmonic frequency components of $37 \mathrm{~Hz}, 88.66 \mathrm{~Hz}, 87 \mathrm{~Hz}$ and signals of two integer harmonic frequency components of $50 \mathrm{~Hz}$, $150 \mathrm{~Hz}$, and no noise. It should be noted that, in this experiment, harmonic components $88.66 \mathrm{~Hz}$, and $87 \mathrm{~Hz}$ with two very similar frequencies were set to verify the estimation ability of the algorithm for inter-harmonics with adjacent frequencies.

The condition was the same as in experiment 1 . For the resulting sequence of samples, the proposed algorithm was used for harmonic frequency estimation, and the estimation result is shown in Fig. (3).

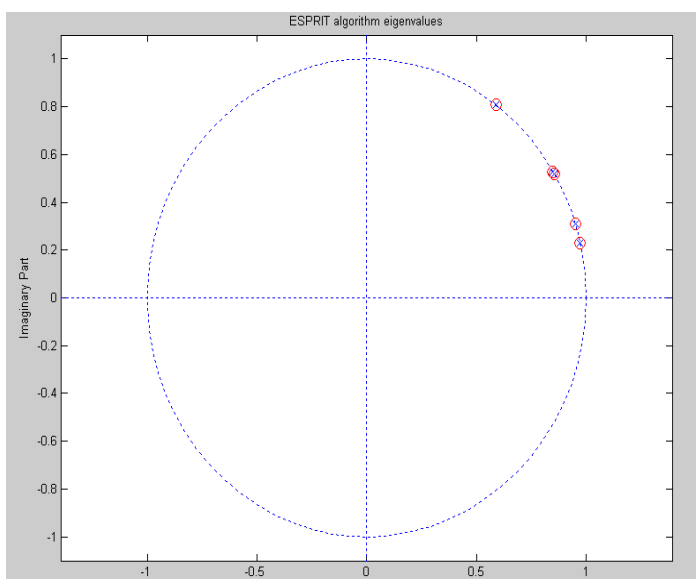

Fig. (3). Experiment 3 time series analysis result.
As the two harmonic frequencies of $88.6 \mathrm{~Hz}$ and $87 \mathrm{~Hz}$ are very close, the local details in the above figure are especially enlarged as in Fig. (4).

For comparison, MUSIC algorithm and FFT algorithm were used under the same condition to estimate the resulting sequence of samples, and the result is shown in Fig. (5) and Table 2.

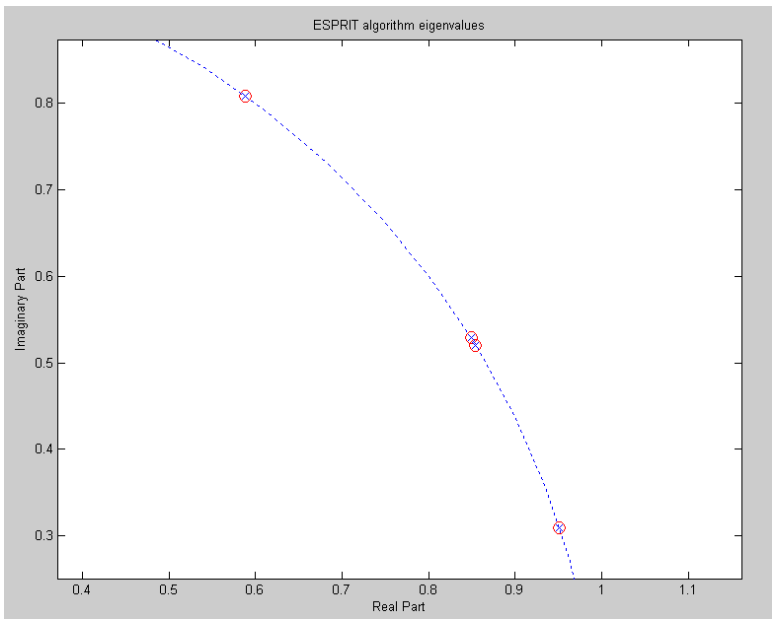

Fig. (4). Partial enlarged drawing of experiment 3.

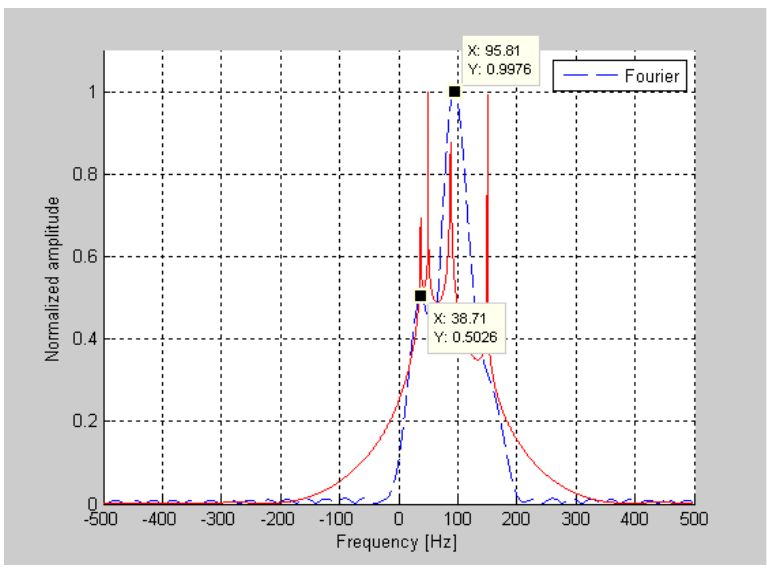

Fig. (5). FFT analysis result of experiment 3. 
It is known, that frequency resolution of FFT algorithm mainly depends on sampling frequency and data amount. In this experiment, $f_{s} / N=33.3 \mathrm{~Hz}$. Due to relatively few sampling points, the resolution was too low to accurately detect each harmonic signal in $x(t)$. Even if there is a peak in the spectrum, the peak number and corresponding frequency cannot correspond to the harmonic number and frequency included in $x(t)$. For comparison, under the same condition, the algorithm discussed here can accurately detect and estimate the frequency of each harmonic signal, which fully demonstrates that the spectral resolving power of this algorithm is far superior to FFT.

Table 2. Experiment 3 harmonic frequency estimation result.

\begin{tabular}{|l|c|c|c|c|c|}
\hline True value (Hz) & 37 & 50 & 87 & 88.66 & 150 \\
\hline ESPRIT estimation & 37.00 & 50.00 & 86.99 & 88.66 & 150 \\
\hline Relative error (\%) & 0 & 0 & 0.01 & 0 & 0 \\
\hline MUSIC estimation & 37.10 & 50.00 & 87.10 & 88.71 & 150 \\
\hline Relative error (\%) & 0.2 & 0 & 0.11 & 0.05 & 0 \\
\hline
\end{tabular}

Using the data in Table 2, the frequency estimation result obtained by the algorithm and standard MUSIC algorithm was compared, and it was found that, the estimation accuracy of the algorithm was similar to that of standard MUSIC algorithm. However, it was noticed that this algorithm avoided characteristic decomposition of sampling data matrix and pseudo-spatial spectrum peak search, therefore its computational complexity was much lower than that of standard MUSIC algorithm.

\section{CONCLUSION}

MUSIC algorithm, one of the most effective methods used for frequency estimation, time delay estimation, and DOA estimation, has extremely high accuracy and is ideally suited for harmonic analysis in electric power. But as the algorithm requires characteristic decomposition of sampling signal autocorrelation matrix, and space spectral peak search in the full frequency domain, its computational complexity is relatively high, which is not conducive to real-time detection of harmonics. This paper combined ESPRIT algorithm based on MSWF fast subspace decomposition technique, and dramatically reduced computational complexity without affecting estimation accuracy. The simulation result shows that the algorithm can achieve estimation of harmonic with ultra-high resolution and has a good anti-noise effect. Such algorithm does not need synchronous sampling, and there is no spectrum leakage problem. Thus, it is very suitable for harmonic analysis in power system, with superiority incomparable to Fourier analysis method.

\section{CONFLICT OF INTEREST}

The authors confirm that this article content has no conflict of interest.

\section{ACKNOWLEDGEMENTS}

Declared none.

\section{REFERENCES}

[1] Q. Jia, X. Yang, and Z. Song, "A windowed frequency shift algorithm for harmonics signal in power system," J. Chin. Electric. Eng. Sci., vol. 36, no. 10, pp. 1631-1640.

[2] T. Lobos, T. Kozina, and H. K. Kozina, "Power system harmonics estimation using linear least squares method and SVD," IEEE Proc. Gen. Trans. Distrib., vol. 148, no. 6, pp. 567-572, 2001.

[3] L. Zhou, X. Xia, and Y. Wan, "Overview of harmonic measurement methods based on wavelet transform," Trans. China Electrotech. Soc., vol. 21, no. 9, pp. 67-74, 2006.

[4] G. Wang, D. Xiang, and W. Ma, "Improved algorithm for noninteger harmonics analysis based on FFT algorithm and neural network," J. Chin. Electric. Eng. Sci., vol. 28, no. 4, pp. 102-108, 2008.

[5] X. Sun, and L. Sun, "MUSIC algorithm for harmonic frequency high resolution estimation in power system," Electrical Measure. Instrum., vol. 10, pp. 28-31, 2008.

[6] X. Wang, and N. Li, "Study on interharmonics detection method based on windowed interpolation FFT algorithm," Modern Power, vol. 29, no. 5, pp. 28-31, 2012.

[7] J. S. Goldstein, I. S. Reed, and L. L. Scharf, "A multistage representation of the wiener filter based on orthogonal projections," IEEE Trans. Inform. Theor., vol. 44, no. 7, pp. 2943-2959, 2002.

[8] R. O. Schmidt, "Multiple emitter location and signal parameter estimation," IEEE Tran. Antennas Propag., vol. 34, no. 3, pp. 276280, 1986.

[9] L. Huang, J. Wu, L. Zhang, and D. Feng, "Rapid subspace decomposition method and rapid estimation of its dimension," Chin. J. Electron., vol. 33, no. 6, pp. 977-981, 2005.

[10] X.D. Zhang, Matrix Analysis and Applications, Tsinghua University Press, China, vol. 9, 2004, pp. 521-524. 